\title{
Primary sclerosing cholangitis in the Swiss Inflammatory Bowel Disease Cohort Study: prevalence, risk factors, and long-term follow-up
}

Montserrat Fraga ${ }^{\mathrm{a}}$, Nicolas Fournier ${ }^{\mathrm{b}}$, Ekaterina Safroneeva ${ }^{\mathrm{d}}$, Valérie Pittet ${ }^{\mathrm{b}}$, Sébastien Godat ${ }^{\mathrm{a}}$, Alex Straumann ${ }^{\mathrm{e}}$, Andreas Nydegger ${ }^{\mathrm{c}}$, Stephan R. Vavricka ${ }^{\mathrm{f}, \mathrm{g}}$, Darius Moradpour ${ }^{\mathrm{a}}$ and Alain M. Schoepfer ${ }^{\mathrm{a}}$; on behalf of the Swiss IBD Cohort Study Group

\begin{abstract}
Background and aim Primary sclerosing cholangitis (PSC) represents the most common hepatobiliary extraintestinal manifestation of inflammatory bowel disease (IBD). We aimed to assess the prevalence of PSC in the Swiss Inflammatory Bowel Disease Cohort Study, to identify associated risk factors, and to describe the long-term evolution.

Patients and methods Data of patients enrolled into the Swiss Inflammatory Bowel Disease Cohort Study were analyzed. Logistic regression modeling was performed to identify risk factors for PSC.

Results Among 2744 patients [1188 ulcerative colitis (UC); 1556 Crohn's disease (CD)], 57 had PSC (48 UC-PSC, nine CDPSC). The prevalence of PSC was higher in UC compared with CD (4.04 vs. $0.58 \%, P<0.001)$. We identified the following significant independent risk factors for PSC in patients with UC: male sex [odds ratio $(\mathrm{OR}) 2.771, P=0.022$ ], pancolitis (OR 2.855, $P=0.011$ ), nonsmoker at diagnosis (OR 9.253, $P=0.030$ ), and a history of appendicectomy (OR 4.114, $P=0.019)$. During a median follow-up time of 74.8 months, four (7.0\%) of PSC patients developed cholangiocarcinoma, six (10.5\%) underwent liver transplantation, and five (8.8\%) died. Survival of IBD-PSC patients was significantly worse compared with IBD patients without PSC $(P=0.001)$. UC-PSC patients developed significantly more frequently colorectal cancer compared with UC patients without PSC (2/48 vs. 9/1440, $P=0.017)$.

Conclusion Approximately $4 \%$ of UC patients and $0.6 \%$ of CD patients had PSC. Male sex, pancolitis, nonsmoker status, and a history of appendicectomy were significantly associated with PSC. PSC is associated with considerable morbidity and mortality in the long term. Eur J Gastroenterol Hepatol 29:91-97

Copyright @ 2016 Wolters Kluwer Health, Inc. All rights reserved.
\end{abstract}

\section{Introduction}

Crohn's disease (CD) and ulcerative colitis (UC), summarized under the term inflammatory bowel diseases (IBD), are chronic inflammatory disorders of the gastrointestinal tract that develop from a dysregulated mucosal immune response to commensal gut flora in genetically susceptible individuals $[1,2]$.

Various extraintestinal manifestations can be associated with IBD [3]. Primary sclerosing cholangitis (PSC) represents the main hepatobiliary extraintestinal manifestations related to IBD. PSC-associated IBD can be found in

\footnotetext{
European Journal of Gastroenterology \& Hepatology 2017, 29:91-97

Keywords: Crohn's disease, inflammatory bowel disease, primary sclerosing cholangitis, ulcerative colitis

aDepartment of Internal Medicine, Division of Gastroenterology and Hepatology, bInstitute of Social and Preventive Medicine, 'Department of Pediatrics, Division of Pediatric Gastroenterology and Hepatology, University Hospital Lausanne and University of, Lausanne, IInstitute of Social and Preventive Medicine, University of Bern, Bern, eDepartment of Internal Medicine, Division of Gastroenterology and Hepatology, University Hospital Basel, Basel, fDepartment of Internal Medicine, Division of Gastroenterology and Hepatology, Stadtspital Triemli and 9Department of Internal Medicine, Division of Gastroenterology and Hepatology, University Hospital Zurich, Zurich, Switzerland

Correspondence to Alain Schoepfer, MD, Division of Gastroenterology and Hepatology, University Hospital Lausanne (CHUV), Rue du Bugnon 44, 1011 Lausanne, Switzerland

Tel: +4121314 0690; fax: +4121314 4718; e-mail: alain.schoepfer@chuv.ch

Received 3 July 2016 Accepted 8 August 2016
}

$60-80 \%$ of affected patients [4]. Conversely, PSC may be observed with a lifetime prevalence of about $5 \%$ of patients with UC and less frequently in patients with CD [5]. PSC is a chronic and progressive disorder characterized by inflammation, fibrosis, and the stricturing processes in the intrahepatic and extrahepatic biliary tree, leading to end-stage liver disease and associated complications such as cholangiocarcinoma [6,7]. Median survival without liver transplantation after PSC diagnosis is 10 to 12 years [8-10]. The exact pathogenesis of PSC is unknown, but environmental as well as genetic factors likely contribute $[11,12]$.

Patients with IBD and concomitant PSC may be characterized by a distinct IBD phenotype that differs from IBD patients without PSC. Pancolitis has been reported to be more prevalent in IBD patients with concomitant PSC compared with IBD patients without PSC [13,14].

Data on the prevalence of PSC, associated risk factors, and natural history from large cohort studies are still limited. Thus, we aimed to answer the following questions using data from the Swiss Inflammatory Bowel Disease Cohort Study (SIBDCS). First, what is the PSC prevalence in all IBD patients and what is the PSC prevalence stratified according to UC and CD? Second, which independent risk factors are associated with PSC in IBD patients? Third, which clinical criteria characterize PSC-IBD patients compared with IBD patients without concomitant PSC? 
Finally, what is the long-term morbidity and mortality of PSC in patients with IBD?

\section{Patients and methods}

\section{Patients}

Since 2006, IBD patients from all regions of Switzerland have been included in the nationwide SIBDCS that is supported by the Swiss National Science Foundation and approved by the local ethical committees [15]. Data acquisition focuses on clinical, socioeconomic, and psychosocial data. Written informed consent is mandatory for inclusion into this cohort as well as permanent residence status in Switzerland or Swiss health insurance. A thorough clinical and laboratory assessment is performed at the time of enrollment. Patients attend follow-up visits at least once a year. The enrollment and follow-up questionnaires capture clinical, socioeconomic, and psychosocial data. Questionnaires assessing the clinical characteristics of IBD patients are completed by the treating physicians, whereas questionnaires that assess, among others, quality of life, social impairment, and symptoms, are completed by IBD patients themselves. Data obtained from patients included in the SIBDCS between July 2006 and May 2014 were analyzed in this study. Patients included in the SIBDCS were recruited in the following healthcare settings: $61 \%$ at university hospitals, $13 \%$ in large nonuniversity hospitals, $6 \%$ in cantonal hospitals, and $20 \%$ in private practices across Switzerland.

\section{Methods}

Data obtained from questionnaires completed by patients and physicians were collected and validated in the data center of the SIBDCS. Rigorous rules were followed to ensure data quality. The diagnosis of PSC was made on the basis of either characteristic alterations of the biliary tree on magnetic resonance cholangiography or endoscopic retrograde cholangiography [16]. The following disorders had to be excluded before PSC was diagnosed: recurrent or chronic bacterial cholangitis, infectious or ischemic cholangiopathy, choledocholithiasis, diffuse intrahepatic metastases, recurrent pancreatitis, IgG4-associated cholangitis, biliary tree alterations after intra-arterial chemotherapy, portal hypertensive biliopathy, and surgical biliary trauma. Liver enzyme abnormalities were not assessed systematically. As information on liver transplantation and PSC-related mortality was not systematically recorded in the questionnaires, the authors contacted the treating physicians of all IBD patients with concomitant PSC to request the following additional information: time of PSC diagnosis, diagnostic modalities, development of cholangiocarcinoma, necessity to undergo liver transplantation related to PSC, and PSC-associated mortality.

Disease location was grouped according to the Montreal classification [17]. For CD, L1 denotes disease in the terminal ileum, L2 denotes disease in the colon, L3 denotes ileocolonic disease, and L4 denotes isolated disease in the upper gastrointestinal tract. For UC, E1 denotes ulcerative proctitis, E2 denotes left-sided colitis, and E3 denotes extensive colitis.

\section{Statistical analysis}

Clinical data were retrieved from the SIBDCS Data Center (Institute of Social and Preventive Medicine, University of Lausanne). All statistical examinations were performed using a statistical package program (STATA, version 12.1; StataCorp., College Station, Texas, USA). Data distribution was analyzed using normal-QQ-plots. Results of the analysis of the quantitative data are presented as either mean \pm SD and range (for Gaussian data) or median and interquartile range (IQR) (for non-Gaussian data). Categorical data were summarized as the percentage of the group total. Differences in distributions of quantitative data were assessed using Student's $t$-test (for Gaussian data) and the Wilcoxon rank-sum test (for non-Gaussian data). Differences between the frequencies for categorical data were assessed using the $\chi^{2}$-test or the Fisher's exact test in case of a small sample size.

To identify factors associated with PSC in IBD, multivariate logistic modeling was performed. First, age at the time of diagnosis, sex, disease location at the time of diagnosis, smoking status at the time of diagnosis, and history of appendicectomy were entered as dichotomous variables, and IBD disease duration was entered as a continuous variable into the univariate logistic regression model. Univariate variables with a $P$-value less than 0.15 were entered into the multivariate logistic model. The results are presented as odds ratio, $95 \%$ confidence interval, and corresponding $P$-values. Survival curves for PSCrelated mortality were calculated according to the Kaplan-Meier method. A $P$-value of less than 0.05 was considered to be statistically significant.

\section{Results \\ Prevalence of PSC in the SIBDCS and clinical characteristics of the PSC-IBD patients}

Among 2744 IBD patients [of whom 1556 (56.7\%) had CD and 1188 (43.3\%) had UC], 57 patients with PSC were identified. Of these, $48(84.2 \%)$ had underlying UC (UC-PSC) and nine (15.8\%) had CD (CD-PSC). The cumulative PSC prevalence was $4.04 \%$ in UC patients and $0.58 \%$ in CD $(P<0.001)$. PSC was significantly more prevalent in men compared with women (risk ratio 2.54, $P=0.001$ ).

The clinical characteristics of UC and CD patients with concomitant PSC are shown in Table 1. Compared with CD-PSC patients, UC-PSC patients were characterized by the following findings: they underwent significantly less frequently intestinal (small bowel) surgery (10.4 vs. $44.4 \%, P=0.027)$ and perianal fistula/abscess surgery $(4.2$ vs. $33.3 \%, P=0.024)$, but they underwent colectomy significantly more frequently (39.6 vs. $0 \%, P=0.022)$. Furthermore, UC-PSC patients were significantly less frequently smoking at IBD diagnosis compared with CD-PSC patients (4.2 vs. $44.4 \%, P<0.001) .5$-Aminosalicylic acid was more frequently used (ever) in UC-PSC patients (85.4 vs. $44.4 \%, P=0.015$ ) compared with CD-PSC patients, whereas immunomodulators were less frequently applied (ever) (54.2 vs. $100 \%, P=0.009$ ). 
$\overline{\text { Table 1. Clinical and demographic characteristics of patients with IBD }}$ with concomitant PSC

\begin{tabular}{|c|c|c|c|}
\hline & Crohn's disease & Ulcerative colitis & $P$-value \\
\hline Number of patients & 9 & 48 & - \\
\hline Male $[n(\%)]$ & $4(44.4)$ & $37(79.3)$ & 0.099 \\
\hline Age at IBD onset (years) & & & 0.930 \\
\hline Median & 23 & 24 & \\
\hline IQR & $21-28$ & $18-35$ & \\
\hline Range & $17-48$ & $10-64$ & \\
\hline IBD duration (years) & & & 0.784 \\
\hline Median & 15 & 13 & \\
\hline IQR & $8-22$ & $7-21$ & \\
\hline Range & $4-37$ & $2-44$ & \\
\hline PSC duration (years) & & & 0.594 \\
\hline Median & 6 & 7 & \\
\hline IQR & $4.5-8$ & $5-13$ & \\
\hline Range & $4-9$ & $3-22$ & \\
\hline BMI $\left(\mathrm{kg} / \mathrm{m}^{2}\right)$ & & & 0.938 \\
\hline Median & 22 & 22 & \\
\hline IQR & $21-23$ & $21-24$ & \\
\hline Range & $18-31$ & $17-48$ & \\
\hline Intestinal surgery $[n(\%)]$ & & & 0.027 \\
\hline No & $5(55.6)$ & $43(89.6)$ & \\
\hline Yes & $4(44.4)$ & $5(10.4)$ & \\
\hline Fistula/abscess surgery $[n(\%)]$ & & & 0.024 \\
\hline No & $6(66.7)$ & $46(95.8)$ & \\
\hline Yes & 3 (33.3) & $2(4.2)$ & \\
\hline Colectomy & & & 0.022 \\
\hline No & $9(100.0)$ & $29(60.4)$ & \\
\hline Yes & $0(0.0)$ & 19 (39.6) & \\
\hline Family history of IBD $[n(\%)]$ & & & 0.790 \\
\hline None & $9(100.0)$ & $39(81.3)$ & \\
\hline Yes & $0(0.0)$ & $4(8.3)$ & \\
\hline Unknown & $0(0.0)$ & $5(10.4)$ & \\
\hline \multicolumn{4}{|c|}{ Smoking status (at diagnosis) $[n(\%)]$} \\
\hline Nonsmoker & $5(55.6)$ & $42(87.5)$ & 0.021 \\
\hline Smoker & $4(44.4)$ & $2(4.2)$ & $<0.001$ \\
\hline Unknown & $0(0.0)$ & $4(8.3)$ & 0.369 \\
\hline Colorectal cancer $[n(\%)]$ & & & 1.000 \\
\hline No & $9(100.0)$ & $46(95.8)$ & \\
\hline Yes & $0(0.0)$ & $2(4.2)$ & \\
\hline EIM other than PSC $[n(\%)]$ & & & 0.146 \\
\hline No & 3 (33.3) & $30(62.5)$ & \\
\hline Yes & $6(66.7)$ & $18(37.5)$ & \\
\hline \multicolumn{4}{|l|}{ History of therapy (ever) $[n(\%)]$} \\
\hline 5-ASA & $4(44.4)$ & $41(85.4)$ & 0.015 \\
\hline Steroids & $7(77.8)$ & $38(79.2)$ & 1.000 \\
\hline Immunomodulators & $9(100.0)$ & $26(54.2)$ & 0.009 \\
\hline Anti-TNF agents & $5(55.6)$ & $18(37.5)$ & 0.461 \\
\hline Calcineurin inhibitors & $1(11.1)$ & $10(20.8)$ & 0.673 \\
\hline
\end{tabular}

5-ASA, 5-aminosalicylic acid; EIM, extraintestinal manifestations; IBD, inflammatory bowel disease; IQR, interquartile range; PSC, primary sclerosing cholangitis; TNF, tumor necrosis factor.

\section{Comparison of UC-PSC patients to UC patients without PSC}

The clinical characteristics and outcomes of the UC-PSC patients $(n=48)$ compared with the UC patients without PSC $(n=1140)$ are shown in Table 2 . The length of the follow-up period was comparable between the groups (69.6 months for UC patients vs. 73.5 months for UC-PSC patients, $P=0.731$ ).

Compared with UC patients without PSC, UC-PSC patients were more frequently men $(77.1$ vs. $53 \%$, $P=0.001$ ), they were younger at UC diagnosis (median 24 vs. 31 years, $P=0.001$ ), they more often had pancolitis (55.3 vs. $37.8 \%, P=0.017$ ), and they more often underwent colectomy ( 39.6 vs. $8.3 \%, P<0.001$ ).

Among the 48 UC-PSC patients, we identified four $(8.3 \%)$ patients with cholangiocarcinoma. In one patient, the cholangiocarcinoma was found only in the explanted
Table 2. Demographic and clinical characteristics of patients with UC with and without PSC

\begin{tabular}{|c|c|c|c|}
\hline $\begin{array}{l}\text { Demographic and clinical } \\
\text { features }\end{array}$ & $\begin{array}{l}\text { UC-PSC } \\
(n=48)\end{array}$ & $\begin{array}{l}\text { UC alone } \\
(n=1140)\end{array}$ & $P$-value \\
\hline Male $[n(\%)]$ & $37(77.1)$ & $604(53)$ & 0.001 \\
\hline \multicolumn{4}{|l|}{ Age at diagnosis of UC (years) } \\
\hline Median & 24 & 31 & 0.001 \\
\hline IQR & $18-35$ & $23-40$ & \\
\hline Range & $10-64$ & $3-82$ & \\
\hline $\begin{array}{l}\text { Disease location at diagnosis } \\
{[n(\%)]}\end{array}$ & & & $<0.001^{*}$ \\
\hline E1 (proctitis) & $1(2.1)$ & $231(20.3)$ & \\
\hline E2 (left-sided colitis) & $10(20.8)$ & $378(33.2)$ & \\
\hline E3 (extensive/pancolitis) & $26(54.2)$ & $432(37.9)$ & \\
\hline $\begin{array}{l}\text { Unknown/unclear location } \\
{[n(\%)]}\end{array}$ & $11(22.9)$ & $99(8.7)$ & \\
\hline Follow-up (months) & & & 0.731 \\
\hline Median & 76.5 & 69.6 & \\
\hline IQR & $44.6-97.6$ & $43.9-93.3$ & \\
\hline Range & $19.6-107.0$ & $2.0-109.3$ & \\
\hline Disease duration (years) & & & 0.005 \\
\hline Median & 13 & 9 & \\
\hline IOR & $7-20.5$ & $5-16$ & \\
\hline Range & $2-44$ & $0-58$ & \\
\hline Colectomy $[n(\%)]$ & $19(39.6)$ & $95(8.3)$ & $<0.001$ \\
\hline Colorectal cancer $[n(\%)]$ & $2(4.2)$ & $9(0.8)$ & 0.017 \\
\hline Cholangiocarcinoma $[n(\%)]$ & 4 (8.3) & NA & NA \\
\hline Liver transplantation [n (\%)] & $6(12.5)$ & NA & NA \\
\hline Mortality $[n(\%)]$ & $5(10.4)$ & $20(1.8)$ & 0.003 \\
\hline
\end{tabular}

Disease location is recorded according to the Montreal classification [17]. IQR, interquartile range; NA, not available; PSC, primary sclerosing cholangitis; UC, ulcerative colitis; UC-PSC, ulcerative colitis associated with primary sclerosing cholangitis.

$P$-values for distinct locations: proctitis, $P=0.002$; left-sided colitis, $P=0.044$; pancolitis, $P=0.017$; unknown disease location, $P<0.001$.

${ }^{*} P$-value for all disease locations together.

liver after transplantation. Of the 48 UC-PSC patients, six $(12.5 \%)$ underwent liver transplantation because of recurrent episodes of cholangitis $(12.5 \%)$. During followup, five $(10.4 \%)$ UC-PSC patients died. The cause of death was cholangiocarcinoma in four patients, whereas one patient died because of advanced liver dysfunction while awaiting liver transplantation. In UC patients without concomitant PSC, the occurrences of cholangiocarcinoma and liver transplantation were not assessed systematically by the cohort questionnaires.

\section{Comparison of CD-PSC patients with CD patients without PSC}

The clinical characteristics and outcomes of the CD-PSC patients compared with the CD patients without PSC are shown in Table 3. Out of 1556 CD patients, a total of nine patients $(0.6 \%)$ had concomitant PSC (CD-PSC). On comparing CD-PSC patients and CD patients without PSC, we observed no statistical difference in the different items. Any comparison between these two groups is limited, given the small number of CD-PSC patients.

\section{Risk factor analysis for PSC in UC patients}

We performed first univariate and then multivariate logistic regression modeling to identify independent risk factors for the occurrence of PSC in UC patients. The results are shown in Table 4. Male sex, nonsmoker status, pancolitis at diagnosis, as well as a history of appendicectomy were found to be associated independently with PSC in UC patients. 
Table 3. Demographic and clinical characteristics of patients with $C D$ with or without PSC

\begin{tabular}{|c|c|c|c|}
\hline Demographic and clinical features & CD-PSC $(n=9)$ & $\begin{array}{l}\mathrm{CD} \text { alone } \\
(n=1547)\end{array}$ & $P$-value \\
\hline Male $[n(\%)]$ & $4(44.4)$ & $733(47.4)$ & 1.000 \\
\hline Age at diagnosis of $C D$ (years) & & & 0.500 \\
\hline Median & 26 & 23 & \\
\hline IQR & $19-36$ & $21-28$ & \\
\hline Range & $4-81$ & $17-48$ & \\
\hline \multicolumn{4}{|l|}{ Disease location at diagnosis $[n(\%)]$} \\
\hline L1 (ileal) & $2(22.2)$ & $353(22.8)$ & \\
\hline L2 (colonic) & $4(44.4)$ & $317(20.5)$ & 0.25 \\
\hline L3 (ileocolonic) & 3 (33.3) & $700(45.3)$ & \\
\hline $\begin{array}{l}\text { L4 (upper gastrointestinal tract) } \\
\text { only }\end{array}$ & 0 & $13(0.8)$ & \\
\hline Unknown/unclear location & 0 & $164(10.6)$ & \\
\hline $\begin{array}{l}\text { Follow-up duration (in the SIBD } \\
\text { Cohort Study) (months) }\end{array}$ & & & 0.103 \\
\hline Median & 101.6 & 72.4 & \\
\hline IQR & $49.6-104.6$ & $45.2-96.5$ & \\
\hline Range & $24.7-107.0$ & $0.6-113.3$ & \\
\hline Disease duration (years) & & & 0.397 \\
\hline Median & 15 & 11 & \\
\hline IQR & $8-22$ & $5-20$ & \\
\hline Range & $4-37$ & $0-55$ & \\
\hline $\begin{array}{l}\text { Small intestine/colonic surgery } \\
{[n(\%)]}\end{array}$ & $1(11.1)$ & $476(38.2)$ & 0.202 \\
\hline Colorectal cancer $[n(\%)]$ & $0(0.0)$ & $4(0.3)$ & 0.879 \\
\hline Cholangiocarcinoma $[n(\%)]$ & 0 & NA & NA \\
\hline Hepatic failure $[n(\%)]$ & 0 & NA & NA \\
\hline Liver transplantation [n (\%)] & 0 & NA & NA \\
\hline Mortality $[n(\%)]$ & 0 & $43(2.8)$ & 0.612 \\
\hline
\end{tabular}

CD, Crohn's disease; CD-PSC, Crohn's disease associated with primary sclerosing cholangitis; IQR, interquartile range; NA, not available; SIBD, Swiss Inflammatory Bowel Disease.

No regression modeling for the identification of risk factors for concomitant PSC was performed in CD patients, given the limited patient number.

\section{Long-term evolution of IBD-PSC patients}

The long-term evolution of IBD-PSC patients was characterized by considerable morbidity and mortality. During a median follow-up time of 74.8 months (6.3 years), $6 / 57(10.5 \%)$ of IBD-PSC patients underwent liver

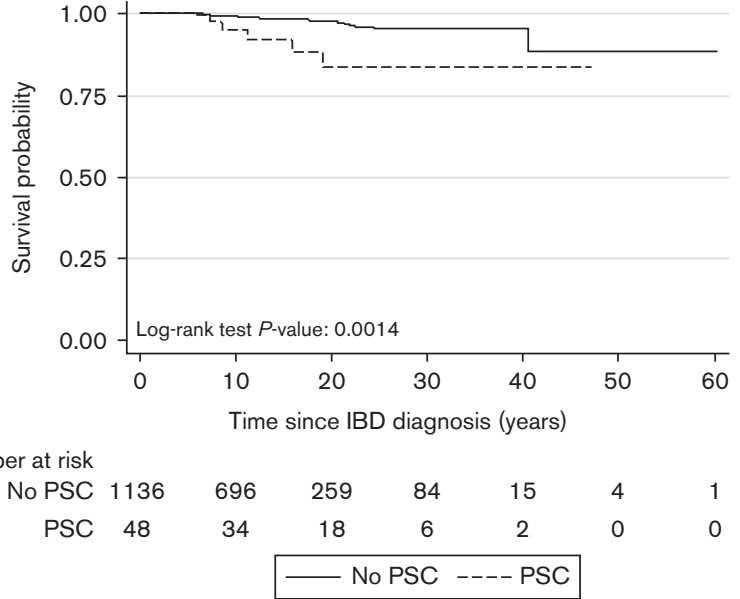

Fig. 1. Cumulative survival of patients with IBD with and without PSC. IBD, inflammatory bowel disease; PSC, primary sclerosing cholangitis.

transplantation because of progressive liver failure and/or recurrent episodes of bacterial cholangitis. All patients who underwent transplantation had underlying UC. In one of the transplanted patients, a cholangiocarcinoma was identified in the explanted liver. The median time interval from PSC diagnosis to diagnosis of cholangiocarcinoma $(n=4)$ was 6 years (IQR $3.5-9$, range $3-10$ years) and 6.5 years (IQR 3-11, range 3-19 years) from PSC diagnosis to liver transplantation $(n=6)$. During the follow-up period, $5 / 57(8.8 \%)$ of IBD-PSC patients died; all of them had underlying UC. Survival of IBD-PSC patients was significantly worse compared with IBD patients without concomitant PSC (Fig. 1, $P=0.001$ ). Mortality in UC-PSC patients during the follow-up period was $10.4 \%$ (5/48) for PSC-UC patients compared with $1.8 \%(20 / 1440)$ for UC patients without concomitant PSC $(P=0.003$, Table 2$)$. No difference in mortality was identified on comparing CD-PSC patients $(n=9)$ with CD patients without concomitant PSC $(n=1547, P=0.612$, Table 3$)$.

All IBD patients with cholangiocarcinoma had UC. A total of $4 / 48(8.3 \%)$ of UC-PSC patients developed

Table 4. Univariate and multivariate logistic regression models to evaluate risk factors for PSC in patients with UC

\begin{tabular}{|c|c|c|c|c|c|c|}
\hline \multirow[b]{3}{*}{ Outcome } & \multicolumn{6}{|c|}{ PSC in patients with UC } \\
\hline & \multicolumn{3}{|c|}{ Univariate model } & \multicolumn{3}{|c|}{ Multivariate model } \\
\hline & OR & 95\% Cl & $P$-value & OR & $95 \% \mathrm{Cl}$ & $P$-value \\
\hline \multicolumn{7}{|l|}{ Age at the time of diagnosis (years) } \\
\hline$<40$ & 1 (ref) & - & - & 1 (ref) & - & - \\
\hline$\geq 40$ & 0.435 & $0.182-1.033$ & 0.059 & 0.568 & $0.210-1.537$ & 0.266 \\
\hline \multicolumn{7}{|l|}{ Sex } \\
\hline Female & 1 (ref) & - & - & 1 (ref) & - & - \\
\hline Male & 2.994 & $1.511-5.917$ & $<0.001$ & 2.771 & $1.154-6.649$ & 0.022 \\
\hline \multicolumn{7}{|l|}{ Pancolitis at diagnosis } \\
\hline No & 1 (ref) & - & - & 1 (ref) & - & - \\
\hline Yes & 3.676 & $1.757-7.692$ & 0.001 & 2.855 & $1.276-6.390$ & 0.011 \\
\hline \multicolumn{7}{|l|}{ Smoking status at the time of diagnosis } \\
\hline Smoker & 1 (ref) & - & - & 1 (ref) & - & - \\
\hline Nonsmoker & 5.682 & $1.364-23.810$ & 0.017 & 9.253 & $1.235-69.341$ & 0.030 \\
\hline Disease duration from the time of diagnosis (years) & 1.040 & $1.012-1.068$ & 0.004 & 1.033 & $0.994-1.074$ & 0.100 \\
\hline \multicolumn{7}{|l|}{ Appendicectomy } \\
\hline No & 1 (ref) & - & - & 1 (ref) & - & - \\
\hline Yes & 3.361 & $1.135-9.954$ & 0.029 & 4.114 & $1.268-13.354$ & 0.019 \\
\hline
\end{tabular}

$\mathrm{Cl}$, confidence interval; OR, odds ratio; $\mathrm{PSC}$, primary sclerosing cholangitis; UC, ulcerative colitis. 
cholangiocarcinoma during the follow-up interval. As such, the annual incidence rate of cholangiocarcinoma in UC-PSC patients was $1.3 \%$.

With a comparable median length of follow-up $(76.5$ vs. 69.6 months), UC-PSC patients developed significantly more frequently a colorectal cancer compared with UC patients without concomitant PSC (2/48 vs. 9/1440, $P=0.017$, Table 2). However, it has to be taken into account that the median disease duration was longer in UC-PSC patients compared with UC patients without concomitant PSC (13 vs. 9 years, $P=0.005)$. A longer disease duration increases the risk of being diagnosed with colorectal cancer.

\section{Discussion}

We present data from the large and well-characterized SIBDCS on the prevalence of PSC, associated risk factors, and the long-term outcome. Our study reports several observations that are clinically relevant: first, PSC is more prevalent in UC than in CD patients. Second, male sex and pancolitis at diagnosis are risk factors for PSC. Third, the mortality of IBD patients with concomitant PSC is significantly higher compared with IBD patients without PSC.

Over a median disease duration of 13 years for UC and 15 years for CD, we observed a PSC prevalence of $4.04 \%$ in UC patients and of $0.58 \%$ in CD patients. Our prevalence data are in accordance with the findings from Olsson et al. [18], who conducted a survey in $1500 \mathrm{UC}$ patients in Sweden. Of these, 72 patients $(4.8 \%)$ were identified to have elevated serum alkaline phosphatase. On the basis of the findings of endoscopic retrograde cholangiopancreatography, which was performed in 65 patients, PSC was diagnosed in 55/1500 UC patients $(3.7 \%)$. Again, similar to our findings, the authors found that PSC was more prevalent in men and more prevalent in UC patients with pancolitis compared with patients with distal colitis [18]. Furthermore, our findings are in line with the data of Broomé et al. [19], who evaluated 1274 UC patients with a follow-up period of 3 years, during which 29 patients $(2.3 \%)$ developed PSC. During the follow-up period, 12 PSC patients died, four because of cholangiocarcinoma and eight because of hepatic failure, whereas one patient underwent liver transplantation [19]. In accordance with our findings, the authors observed an increased mortality in UC patients with concomitant PSC compared with patients with UC without PSC. Given the longer follow-up period, it is to be expected that the PSC prevalence in the SIBDCS is slightly higher compared with the data from Broomé and colleagues.

In our cohort, PSC prevalence in CD patients $(0.58 \%)$ was clearly lower compared with UC patients $(4.04 \%)$. Despite a comparable follow-up time of 15 years, the PSC prevalence in $\mathrm{CD}$ patients is lower than that reported by Rasmussen et al. [20], who diagnosed PSC, on the basis of liver enzyme abnormalities and compatible findings in endoscopic retrograde cholangiopancreatography, in $9 / 262(3.4 \%)$ of CD patients. It is noteworthy that all PSCaffected $\mathrm{CD}$ patients in that study had colonic involvement [20].

We carried out an extensive analysis on potential associated factors for PSC development in UC patients by means of logistic regression modeling. We identified male sex, pancolitis at UC diagnosis, nonsmoker status at diagnosis, as well as a history of appendicectomy as factors significantly associated with a diagnosis of PSC. Our data confirm the findings of Joo et al. [21], who previously reported that UC patients with PSC had more frequently extensive disease and pancolitis, when compared with UC patients without concomitant PSC. In addition, we were also able to reproduce the already published evidence that male sex is a risk factor for PSC development among UC patients [22]. We found cigarette smoking at UC diagnosis to be a significant independent protective factor for PSC development (odds ratio $0.12, P=0.040$ ). The protective effect of cigarette smoking on PSC development was already shown two decades ago [23,24]. Controversial evidence is found in the literature on the impact of appendicectomy on the risk of PSC development. In our cohort, a history of appendicectomy was an independent significant risk factor for PSC development. This finding is not in line with data from Florin et al. [25] as well as Mitchell et al. [26], who did not find that appendectomy increased the risk for PSC. As a word of caution, we need to mention that the SIBDCS questionnaires did not assess the exact year of appendicectomy. As such, the temporal relationship between appendicectomy and PSC diagnosis remains unclear.

The long-term outcome of IBD patients with PSC in the SIBDCS was characterized by considerable morbidity and mortality. Survival of IBD-PSC patients was significantly worse compared with IBD patients $(P=0.001)$. During a follow-up period of 6.3 years, $8.3 \%$ (4/48) of UC-PSC patients developed cholangiocarcinoma, which results in an annual incidence rate of $1.3 \%$. As such, our data are in accordance with the results from Bergquist et al. [27], who determined in a large cohort study comprising 604 Swedish PSC patients an annual incidence rate of cholangiocarcinoma of $1.5 \%$. The Swiss data on the considerable morbidity and mortality associated with PSC are also in accordance with findings from Tischendorf et al. [28], who described a German cohort of 273 PSC patients over a median follow-up time of 76 months (which compares well with the follow-up time in the SIBDCS). The authors found that the estimated median survival from the time of diagnosis to death or time of liver transplantation was 9.6 years. In the German cohort, 108 (39.6\%) patients underwent liver transplantation and hepatobiliary malignancies were found in $39(14.3 \%)$ patients.

Our study does have strengths as well as some limitations. The data stem from the large and well-characterized SIBDCS. We confirm the data of previous cohort studies reporting on PSC prevalence in UC and CD, associated risk factors, as well as the significantly increased mortality of IBD patients affected by PSC. A detailed hand search of source documents (patient files) was performed in IBD patients with concomitant PSC to seek information on items that are not routinely captured by the SIBDCS questionnaires. One important limitation is that our national cohort is not population based. As such, IBD patients recruited in hospitals $(80 \%)$ are over-represented compared with those recruited from private practice $(20 \%)$. The results may therefore not be applicable to all IBD patients. Second, we cannot provide incidence data on PSC in the IBD population. Third, the evaluated risk factors for CD-PSC patients are not solid enough for 
interpretation, given the small sample size $(n=9$ CD-PSC patients). Fourth, the observed disease duration of UC-PSC patients (median 13 years) was significantly longer compared with the disease duration of UC alone patients (median 9 years, $P=0.005$ ), which increases the risk for undergoing complications.

In summary, we could confirm previously published data on PSC prevalence, associated risk factors, and the unfavorable long-term evolution in a large and wellcharacterized cohort of IBD patients. Our data emphasize the need to pay particular attention during the follow-up of IBD patients with concomitant PSC.

\section{Acknowledgements}

The authors thank all members of the SIBDCS who were involved in the acquisition of data: Claudia Anderegg; Peter Bauerfeind; Christoph Beglinger; Stefan Begré; Dominique Belli; José Bengoa; Luc Biedermann; Janek Binek; Mirjam Blattmann; Nadia Blickenstorfer; Stephan Boehm; Jan Borovicka; Christian Braegger; Patrick Bühr; Bernard Burnand; Emmanuel Burri; Sophie Buyse; Matthias Cremer; Dominique Criblez; Philippe de Saussure; Lukas Degen; Joakim Delarive; Christopher Dörig; Barbara Dora; Gian Dorta; Tobias Ehmann; Ali El Wafa; Mara Egger; Matthias Engelmann; Christian Felley; Markus Fliegner; Nicolas Fournier; Montserrat Fraga; Alain Frei; Pascal Frei; Remus Frei; Michael Fried; Florian Froehlich; Raoul Furlano; Suzanne Gallot-Lavallée; Martin Geyer; Marc Girardin; Delphine Golay; Tanja Grandinetti; Beat Gysi; Horst Haack; Johannes Haarer; Beat Helbling; Peter Hengstler; Denise Herzog; Cyrill Hess; Klaas Heyland; Thomas Hinterleitner; Philippe Hiroz; Claudia Hirschi; Petr Hruz; Pascal Juillerat; Rosmarie Junker; Christina Knellwolf; Christoph Knoblauch; Henrik Köhler; Rebekka Koller; Claudia Krieger; Gerd A. Kullak-Ublick; Markus Landolt; Frank Lehmann; Valérie McLin; Philippe Maerten; Michel Maillard; Christine Manser; Urs Marbet; Michael Manz; George Marx; Rémy Meier; Christa Meyenberger; Jonathan Meyer; Pierre Michetti; Benjamin Misselwitz; Darius Moradpour; Patrick Mosler; Christian Mottet; Christoph Müller; Pascal Müller; Beat Müllhaupt; Claudia Münger; Leilla Musso; Andreas Nagy; Cristina Nichita; Natacha Noël; Andreas Nydegger; Maliza Nzabonimpa; Nicole Obialo; Carl Oneta; Cassandra Oropesa; LaetitiaMarie Petit; Franziska Piccoli; Julia Pilz; Gaëlle Pittet; Valérie Pittet; Bruno Raffa; Ronald Rentsch; Sophie Restellini, Jean-Pierre Richterich; Silvia Rihs; Jocelyn Roduit; Daniela Rogler; Gerhard Rogler; Jean-Benoît Rossel; Markus Sagmeister; Gaby Saner; Bernhard Sauter; Mikael Sawatzki; Michael Scharl; Sylvie Scharl; Nora Schaub; Martin Schelling; Susanne Schibli; Hugo Schlauri; Daniela Schmid; Sybille Schmid; Jean-François Schnegg; Alain Schoepfer; Christiane Sokollik; Frank Seibold; GianMarco Semadeni; Mariam Seirafi; David Semela; Arne Senning; Marc Sidler; Johannes Spalinger; Holger Spangenberger; Philippe Stadler; Volker Stenz; Michael Steuerwald; Alex Straumann; Michael Sulz; Alexandra Suter; Michela Tempia-Caliera; Joël Thorens; Sarah Tiedemann; Stephan Vavricka; Francesco Viani; Roland Von Känel; Alain Vonlaufen; Dominique Vouillamoz;
Rachel Vulliamy; Helene Werner; Paul Wiesel; Reiner Wiest; Tina Wylie; Jonas Zeitz; Dorothee Zimmermann.

This work was supported by grants from the Swiss National Science Foundation (33CS30_148422 to Swiss IBD Cohort Study group, 32003B_135664/1 to A.M.S., 320000-114009/3 and 32473B_135694/1 to S.R.V.) and unrestricted research grants from Otsuka Switzerland, Takeda Switzerland, and Tillotts Switzerland. This is an investigator-initiated study; pharmaceutical companies played no role in study design, acquisition, analysis, interpretation, or presentation of the data.

\section{Conflicts of interest}

There are no conflicts of interest.

\section{References}

1 Van Assche G, Dignass A, Panes J, Beaugerie L, Karagiannis J, Allez M, et al. The second European evidence-based Consensus on the diagnosis and management of Crohn's disease: definitions and diagnosis. J Crohns Colitis 2010; 4:7-27.

2 Dignass A, Eliakim R, Magro F, Maaser C, Chowers Y, Geboes K, et al. Second European evidence-based consensus on the diagnosis and management of ulcerative colitis part 1: definitions and diagnosis. J Crohns Colitis 2012; 6:965-990.

3 Ardizzone S, Puttini PS, Cassinotti A, Porro GB. Extraintestinal manifestation of inflammatory bowel disease. Dig Liver Dis 2008; 40:253-259.

4 Rossi RE, Conte D, Massironi S. Primary sclerosing cholangitis associated with inflammatory bowel disease: an update. Eur J Gastroenterol Hepatol 2016; 28:123-131.

5 Kaplan GG, Laupland KB, Butzner D, Urbanski SJ, Lee SS. The burden of large and small duct primary sclerosing cholangitis in adults and children: a population-based analysis. Am J Gastroenterol 2007; 102:1042-1049.

6 LaRusso NF, Wiesner RH, Ludwig J, MacCarty RL. Current concepts. Primary sclerosing cholangitis. N Engl J Med 1984; 310:899-903.

7 Rosen CB, Nagomery DM, Wiesner RH, Coffey RJ Jr, LaRusso NF. Cholangiocarcinoma complicating primary sclerosing cholangitis. Ann Surg 1991; 213:21-25.

8 Tischendorf JJ, Hecker $H$, Krüger M, Manns MP, Meier PN. Characterization, outcome, and prognosis in 273 patients with primary sclerosing cholangitis: a single center study. Am J Gastroenterol 2007; 102:107-114.

9 Wiesner $\mathrm{RH}$, Grambsch PM, Dickson ER, Ludwig J, MacCarty RL, Hunter EB, et al. Primary sclerosing cholangitis: natural history, prognostic factors and survival analysis. Hepatology 1989; 10:430-436.

10 Broomé U, Olsson R, Lööf L, Bodemar G, Hultcrantz R, Danielsson A, et al. Natural history and prognostic factors in 305 Swedish patients with primary sclerosing cholangitis. Gut 1996; 38:610-615.

11 Melum E, Franke A, Schramm C, Weismüller TJ, Gotthardt DN, Offner FA, et al. Genome-wide association analysis in primary sclerosing cholangitis. Nat Genet 2011; 43:17-19.

12 Janse M, Lamberts LE, Franke L, Raychaudhuri S, Ellinghaus E, Muri Boberg K, et al. Three ulcerative colitis susceptibility loci are associated with primary sclerosing cholangitis and indicate a role for IL2, REL, and CARD9. Hepatology 2011; 53:1977-1985.

13 Boonstra K, van Erpecum KJ, van Nieuwkerk KM, Drenth JP, Poen AC, Witteman BJ, et al. Primary sclerosing cholangitis is associated with a distinct phenotype of inflammatory bowel disease. Inflamm Bowel Dis 2012; 18:2270-2276.

14 Loftus EV Jr, Harewood GC, Loftus CG, Tremaine WJ, Harmsen WS, Zinsmeister AR, et al. PSC-IBD: a unique form of inflammatory bowel disease associated with primary sclerosing cholangitis. Gut 2005; 54:91-96.

15 Pittet V, Juillerat P, Mottet C, Felley C, Ballabeni P, Burnand B, et al. Cohort profile: the Swiss Inflammatory Bowel Disease Cohort Study (SIBDCS). Int J Epidemiol 2009; 38:922-931.

16 Lindor KD, Kowdley KV, Harrison ME. ACG clinical guideline: primary sclerosing cholangitis. Am J Gastroenterol 2015; 110:646-659; quiz 660. 
17 Silverberg MS, Satsangi J, Ahmad T, Arnott ID, Bernstein CN, Brant SR, et al. Toward an integrated clinical, molecular and serological classification of inflammatory bowel disease: report of a Working Party of the 2005 Montreal World Congress of Gastroenterology. Can J Gastroenterol 2005; 19 (Suppl A):5A-36A.

18 Olsson R, Danielsson A, Järnerot G, Lindström E, Lööf L, Rolny P, et al. Prevalence of primary sclerosing cholangitis in patients with ulcerative colitis. Gastroenterology 1991; 100 (Pt 1):1319-1323.

19 Broomé U, Glaumann H, Hellers G, Nilsson B, Sörstad J, Hultcrantz R. Liver disease in ulcerative colitis: an epidemiological and follow up study in the county of Stockholm. Gut 1994; 35:84-89.

20 Rasmussen $\mathrm{HH}$, Fallingborg JF, Mortensen PB, Vyberg M, TageJensen U, Rasmussen SN. Hepatobiliary dysfunction and primary sclerosing cholangitis in patients with Crohn's disease. Scand $J$ Gastroenterol 1997; 32:604-610.

21 Joo M, Abreu-e-Lima P, Farraye F, Smith T, Swaroop P, Gardner L, et al. Pathologic features of ulcerative colitis in patients with primary sclerosing cholangitis: a case-control study. Am J Surg Pathol 2009; 33:854-862.

22 Gizard E, Ford AC, Bronowicki JP, Peyrin-Biroulet L. Systematic review: the epidemiology of the hepatobiliary manifestations in patients with inflammatory bowel disease. Aliment Pharmacol Ther 2014; 40:3-15.
23 Loftus EV Jr, Sanborn WJ, Tremaine WJ, Mahoney DW, Zinsmeister AR, Offord KP, et al. Primary sclerosing cholangitis is associated with non smoking: a case-control study. Gastroenterology 1996; 110:1496-1502.

24 Van Erpecum KJ, Smits SJ, van de Meeberg PC, Linn FH, Wolfhagen $\mathrm{FH}$, vanBerge-Henegouwen GP, Algra A. Risk of primary sclerosing cholangitis is associated with nonsmoking behavior. Gastroenterology 1996; 110:1503-1506.

25 Florin TH, Pandeya N, Radford-Smith GL. Epidemiology of appendicectomy in primary sclerosing cholangitis and ulcerative colitis: its influence on the clinical behaviour of these diseases. Gut 2004; 53:973-979.

26 Mitchell SA, Thyssen M, Orchard TR, Jewell DP, Fleming KA, Chapman RW. Cigarette smoking, appendectomy, and tonsillectomy as risk factors for the development of primary sclerosing cholangitis: a case-control study. Gut 2002; 51:567-573.

27 Bergquist A, Ekbom A, Olsson R, Kornfeldt D, Lööf L, Danielsson A, et al. Hepatic and extrahepatic malignancies in primary sclerosing cholangitis. J Hepatol 2002; 36:321-327.

28 Tischendorf JJ, Meier PN, Strassburg CP, Klempnauer J, Hecker H, Manns MP, Krüger M. Characterization and clinical course of hepatobiliary carcinoma in patients with primary sclerosing cholangitis. Scand $J$ Gastroenterol 2006; 41:1227-1234. 